\title{
Correction to: One hour post-load plasma glucose and 3 year risk of worsening fasting and 2 hour glucose tolerance in the RISC cohort
}

\author{
Melania Manco ${ }^{1} \cdot$ Andrea Mari $^{2} \cdot$ John $^{\text {Petrie }}{ }^{3} \cdot$ Geltrude Mingrone $^{4,5,6} \cdot$ Beverley Balkau $^{7} \cdot$ for the EGIR-RISC study \\ group
}

Published online: 21 March 2019

(C) Springer-Verlag GmbH Germany, part of Springer Nature 2019

\section{Correction to: Diabetologia}

https://doi.org/10.1007/s00125-018-4798-5

The affiliation details for Geltrude Mingrone are corrected below.

Publisher's note Springer Nature remains neutral with regard to jurisdictional claims in published maps and institutional affiliations.

The online version of the original article can be found at https://doi.org/ $10.1007 / \mathrm{s} 00125-018-4798-5$

\footnotetext{
Melania Manco

melania.manco@opbg.net

john.petrie@glasgow.ac.uk
}

$\triangle$ John Petrie

1 Research Area for Multifactorial Diseases and Complex Phenotypes, Bambino Gesù Children's Hospital, Viale Ferdinando Baldelli 38, 00146 Rome, Italy

2 CNR Institute of Neuroscience, Padua, Italy

3 Institute of Cardiovascular and Medical Sciences, BHF Glasgow Cardiovascular Research Centre, University of Glasgow, 126 University Place, Glasgow G12 8TA, UK

4 Fondazione Policlinico Universitario A. Gemelli IRCCS, Roma, Italia

5 Università Cattolica del Sacro Cuore, Roma, Italia

6 Department of Diabetes, King's College London, London, UK

7 CESP Centre for Research in Epidemiology and Population Health, Univ Paris-Saclay, Univ Paris Sud, Villejuif, France 